\title{
Parametric Point Estimation of the Geeta Distribution
}

\section{Betty Korir}

Department of Mathematics \& Computer Science, University of Eldoret, Eldoret, Kenya

\section{Email address:}

bettykorir2006@yahoo.com

\section{To cite this article:}

Betty Korir. Parametric Point Estimation of the Geeta Distribution. International Journal of Statistical Distributions and Applications. Vol. 4, No. 3, 2018, pp. 51-59. doi: 10.11648/j.jisd.20180403.11

Received: September 28, 2018; Accepted: November 2, 2018; Published: November 29, 2018

\begin{abstract}
Geeta distribution is a new discrete random variable distribution defined over all the positive integers with two parameters. This distribution belongs to the family of Location-parameter (LDPD) system and is of the form L - shaped model. Pareto and Yule distributions belong to the same family but these distributions have a disadvantage of having a single parameter which makes them not versatile to meet the needs of modern complex data sets. Geeta distribution is found to be very versatile and flexible to fit observed count data sets and can be used efficiently to model different types of sets. This paper investigates the characteristics of Geeta distribution, such as the existence of the mean, variance, moment generating function, probability generating function and that the sum of probabilities for all values of $\mathrm{X}$ for Geeta Distribution model is unity. It is well known that the sample mean is the estimator of a population mean from a given population of interest as a point estimator which assume a single number that is obtained by taking a random sample of a specified size from the entire population, depending on whether the population mean and variance is known or unknown These point estimators were obtained by employing the method of Moments, Maximum Likelihood (MLE) and Bayesian estimator. Further the estimators were subjected to the conditions like unbiasedness, efficiency, sufficiency and completeness which are properties of a good estimator. For the first aspect, the results of the mean, variance, moments and generating functions were achieved that proves the distribution is a probability density function (pdf). The methods of moments and the maximum likelihood and their properties were applied and yielded the desired and expected results for any given probability distribution. The best estimator obtained is best linear unbiased estimator (BLUE).
\end{abstract}

Keywords: Geeta Distribution, Maximum Likelihood Estimators, Methods of Moments

\section{Introduction}

The estimation of parameters of a probability distribution is a problem of basic importance in modern mathematical statistics [1]. However, several theories of estimation are available which apply under general conditions and are categorized into two broad classes, the parametric interval estimation and parametric point estimation [2]. This paper focuses on the parametric point estimation and any given distribution expressed in the mathematical form either in its discrete or continuous state, the techniques of obtaining the estimators shall apply.

Given a population with a probability density function $\mathrm{f}\left(\mathrm{x}_{\mathrm{i}}\right.$, $\left.\theta_{1}, \theta_{2}, \ldots, \theta_{n}\right)$ which would completely be determined if the values of the parameters $\theta_{1}, \theta_{2}, \ldots, \theta_{n}$ were known. Let $X_{1}$, $\mathrm{X}_{2} \ldots, \mathrm{X}_{\mathrm{n}}$ be a random sample of observations $\mathrm{X}_{1}, \mathrm{X}_{2}, \ldots, \mathrm{X}_{\mathrm{n}}$ from this population, it is required to determine some function $t\left(\mathrm{X}_{1}, \mathrm{X}_{2} \ldots, \mathrm{X}_{\mathrm{n}}\right)$, such that the distribution of $t\left(\mathrm{X}_{1}\right.$, $\left.\mathrm{X}_{2} \ldots, \mathrm{X}_{\mathrm{n}}\right)=t(\mathrm{x})$ upon repeated sampling from the same population, with samples of equal size, $n$ will be concentrated around the values of the parameters of the population [2].

The function of a random sample $\mathrm{X}_{1}, \mathrm{X}_{2}, \ldots \mathrm{X}_{\mathrm{n}}$ is called the statistics, $t(\mathrm{x})$ and the values it assumes with each particular random sample are called estimators. There are Several properties that a statistics, $t(\mathrm{x})$ should satisfy in order to qualify as a good estimator, that is an estimator with a distribution closely concentrated around the value of $\theta_{1}, \theta_{2}, \ldots$, $\theta_{\mathrm{n}}$, should be unbiased,consistent,efficient,sufficient and complete statistic.

\subsection{The Geeta Distribution}

Geeta distribution is a newly introduced distribution which has two unknown parameters and is of the form L-shaped 
model, it belongs to a family of Modified Power Series distribution(MPSD), the langrangian series distributions and location parameter distribution. The Yule distribution and Pareto which belongs to the same family(MPSD) have a single parameter but fails the test of handling large data sets when it comes to applications in modern technologies [3]. Geeta distribution model is very versatile in meeting the needs of modern complex data sets and this is attributed to the presence of the two unknown constants when compared to the distribution of the same class. The unknown constants can be estimated using the estimation techniques and it is believed that these constants contain a lot of information since estimation is accompanied by statistical inference.

Geeta distribution is defined as a discrete random variable, $\mathrm{X}$, over the set of all positive integers, with the probability mass function [4] given by

$$
\begin{aligned}
& \operatorname{Pr}(X=x)= \\
& \frac{1}{\beta x-1}\left(\begin{array}{c}
\beta x-1 \\
x
\end{array}\right) \theta^{x-1}(1-\theta)^{\beta x-x} ; x=1,2,3, \cdots \\
& \quad=0, \text { otherwise }
\end{aligned}
$$

where, $0<\theta<1 ; 1<\beta<\theta^{-1}$

The upper limit on $\beta$ has been imposed for the existence of the mean. When $\beta \rightarrow 1$ the model degenerates to a single point at $\mathrm{x}=1$.

The Geeta distribution has a maximum as $\mathrm{x}=1$ and is $\mathrm{L}-$ shaped for all values of $\theta$ and $\beta$. It may have a short tail or a long tail and heavy tail depending upon the values of $\theta$ and $\beta$. Its mean, $\mu$ and variance $\sigma^{2}$ are given by

$$
\mu=(1-\theta)(1-\beta \theta)^{-1}
$$

and

$$
\sigma^{2}=(\beta-1) \theta(1-\theta)(1-\beta \theta)^{-3}
$$

And from the formula (2), $\theta=\frac{(\mu-1)}{(\beta \mu-1)}$.

The family of Geeta probability model belongs to the classes of the modified power series distribution (MPSD) and the Langrangian series distribution. It can also be expressed as a location parameter probability distribution [3] given below

$$
\begin{aligned}
& \operatorname{Pr}(X=x)=\frac{1}{\beta x-1}\left(\begin{array}{c}
\beta x-1 \\
x
\end{array}\right)\left(\frac{\mu-1}{\beta \mu-1}\right)^{x-1}\left(\frac{\mu(\beta-1}{\beta \mu-1}\right)^{\beta x-x} \\
& ; x=1,2,3, \cdots \\
& \quad=0, \text { otherwise }
\end{aligned}
$$

where $\mu$ is the mean and $\beta>1$. Note that this form does not have an upper limit on $\beta$. Consul (1990) has shown that the Geeta distribution (4) can be characterized by its variance:

$$
\sigma^{2}=(\beta-1)^{-1} \mu(\mu-1)(\beta \mu-1)
$$

and the domain of $\mathrm{X}$.

It is clear from the expression of $\sigma^{2}$ that $\frac{d \sigma^{2}}{d \mu}>0$ and accordingly, $\sigma^{2}$ increases monotonically as increases in value and that the smallest value of $\sigma^{2}$ is zero when $\mu=1$ that is, when the model reduces to a single point $\mathrm{x}=1$.

$$
\text { Also, } \frac{d \sigma^{2}}{d \beta}=\frac{-\mu(\mu-1)^{2}}{(\beta-1)^{2}}<0
$$

Thus, the variance $\sigma^{2}$ decreases monotonically as $\beta$ increases and the smallest value of $\sigma^{2}$, for the largest value of $\beta$ becomes $\mu^{2}(\mu-1)$. From this we conclude that when $\beta \mu-1 \leq(\beta-1)(\mu-1)^{-1}$

the variance will be less than the mean, $\mu$ and will have the range:

$$
\mu^{2}(\mu-1)<\sigma^{2} \leq \mu
$$

If $(\beta \mu-1)>(\beta-1)(\mu-1)^{-1}$ the value of $\sigma^{2}$ will become larger than $\mu$.

\subsection{Bar Diagrams for Geeta Distribution Model}

The successive probabilities for various values of $X$ can be easily computed from the values:

$$
\begin{aligned}
& \operatorname{Pr}(X=1)=\left[\frac{(\beta-1) \mu}{\beta \mu-1}\right]^{\beta-1}, \\
& \operatorname{Pr}(X=2)=\frac{\mu-1}{\mu}\left[\frac{(\beta-1) \mu}{\beta \mu-1}\right]^{2 \beta-1}, \cdots
\end{aligned}
$$

and the recurrence formula

$$
\begin{aligned}
& \operatorname{Pr}(X=K+1)= \\
& \underset{i=1}{\bigsqcup}\left[1+\frac{\beta}{k \beta-i}\right] \frac{\mu-1}{\mu}\left[\frac{(\beta-1)}{\beta \mu-1}\right]^{\beta} \operatorname{Pr}(X=k)
\end{aligned}
$$

for $\mathrm{k}=2,3,4$,?

The probabilities for the Geeta distribution (4) were computed for $\mu=1.2$ to 5.2 varying by 0.2 and for values of $\beta$ varying from 1.2 to 4.2 (with increments of 0.6 ) and bardiagrams were drawn for all of them to see the variations. Twelve of these bar-diagrams are shown above for two typical values of $\mu=1.2,5.2$ and for $\beta=1.2,1.8,2.4,3.0$, 3.6, 4.2 corresponding to each values of $\mu$. 


\subsubsection{Bar-Diagram for Geeta Model for $\mu=1.2$}
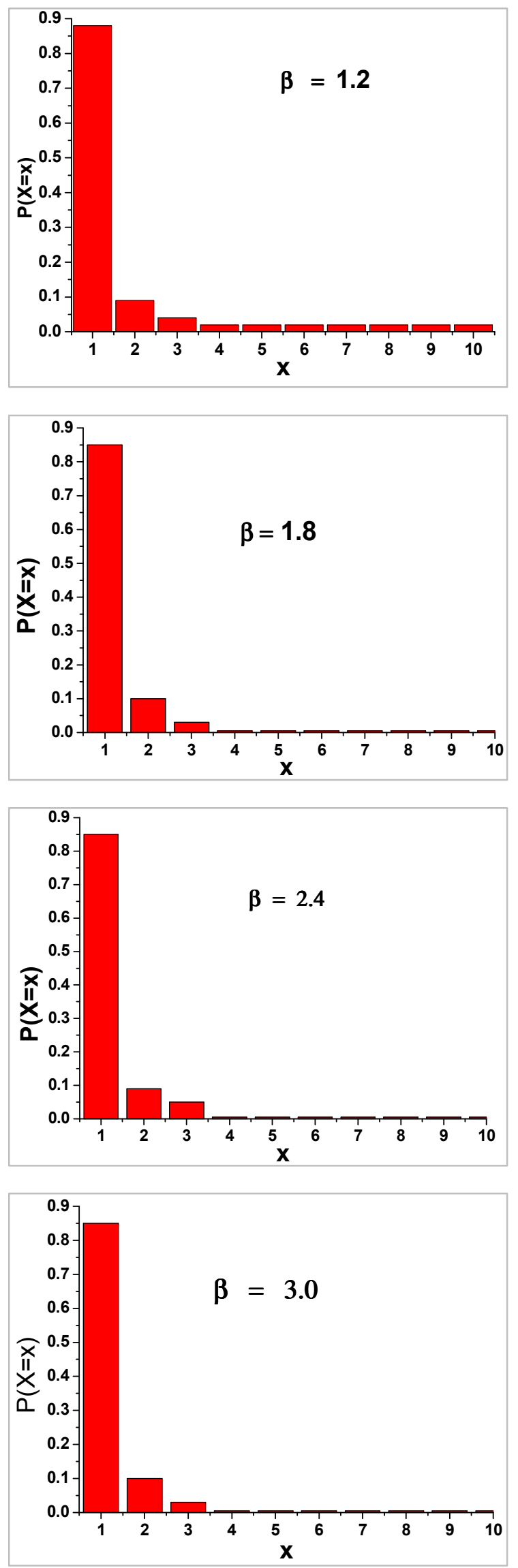


Figure 1. Probabilities for values of $X$ at $\mu=1.2$ for different values of $\beta$ [2] [1].

\subsubsection{Bar-Diagram for Geeta Model for $\mu=5.2$}
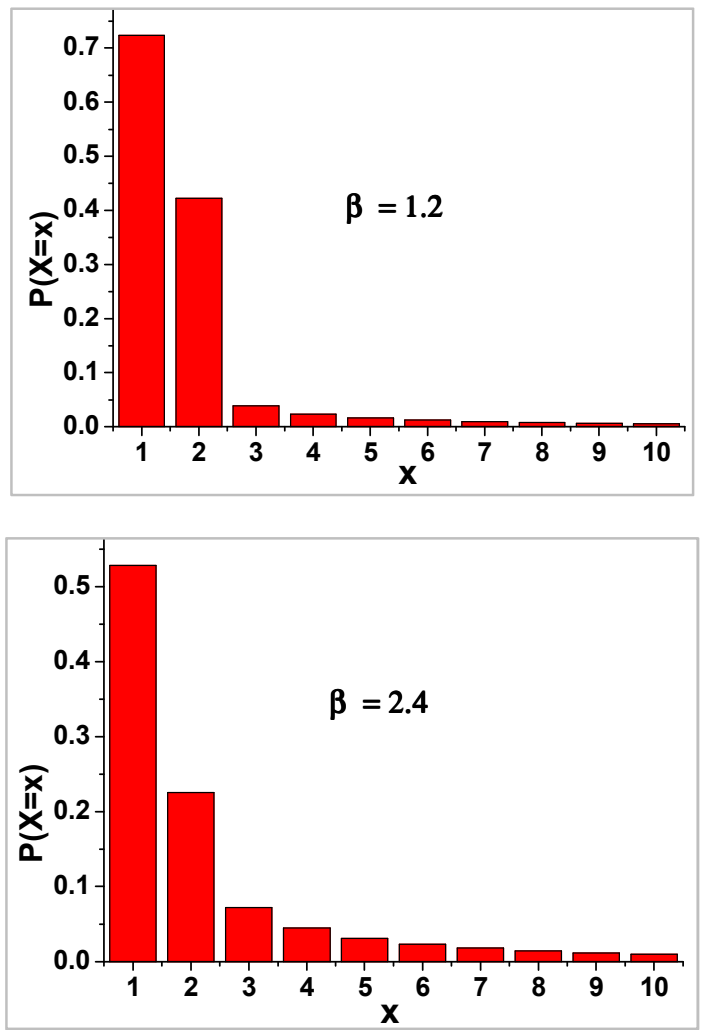

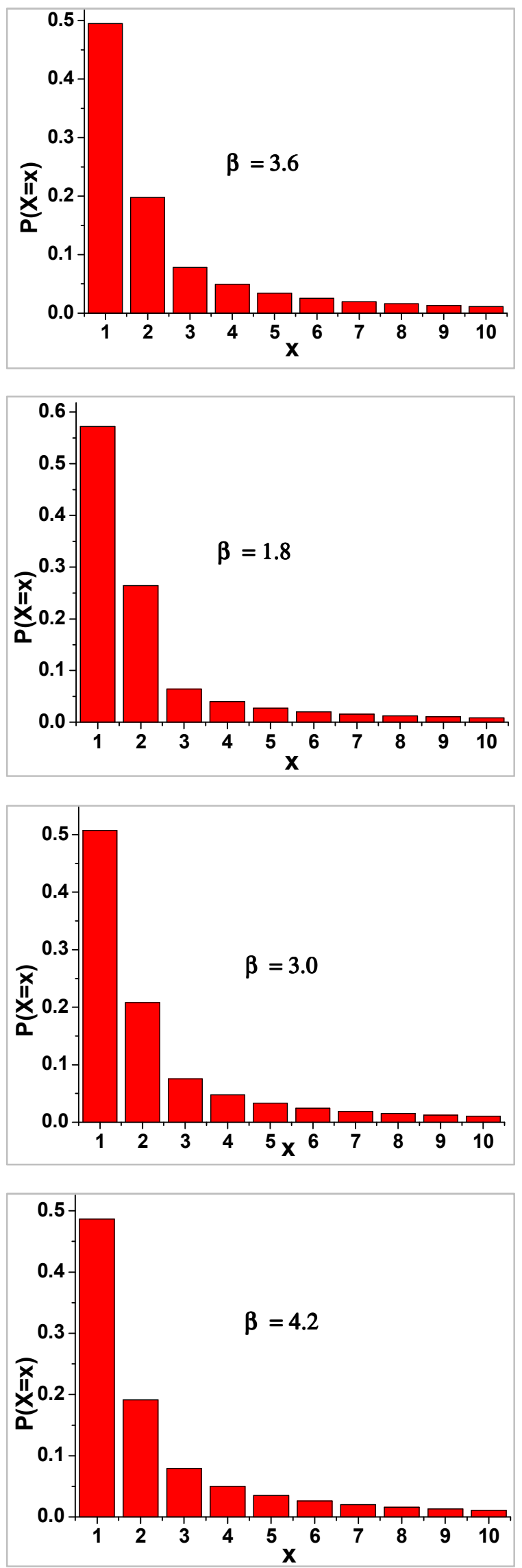

Figure 2. Probabilities for values of $X$ at $\mu=5.2$ for different values of $\beta$ [2].
It is clear from these graphs that $\operatorname{Pr}(X=1)$ reduces as $\mu$ increases and the probabilities for all other values of $x$ increase but the model always remains L-shaped. Thus the tail becomes more and more heavy and longer with the increase in the value of $\mu$. There is a similar effect when the value of $\mu$ is kept fixed and the value of $\beta$ is slowly increased. The value of $\operatorname{Pr}(X=1)$ decreases and the probabilities for other values of $\mathrm{x}$ increase as $\beta$ increases. However, these changes for $\beta$ are at a much slower pace than the changes for $\mu$ with the result that the Geeta probability model becomes more suitable and versatile than some other models for abundance data sets.

\section{Methodology}

The parametric point estimation is twofold; finding point estimators and then determining good, or optimum estimator. In the preliminaries stages a statistic is obtained by taking a sample of size, $n$ from the entire population. A statistic can be expressed a function of sample observation and there are many statistics from same population that can be obtained. Assume that some characteristics of the elements in a population can be represented by a random variable $\mathrm{X}$ whose density is $f_{X}(\cdot ; \theta)$, where the form of the density is assumed known except that it contains an unknown parameter $\theta$. Further assume that the values $x_{1}, x_{2}, \ldots, x_{n}$ of a random sample $X_{1}, X_{2}, \cdots, X_{n}$ from $f_{X}(\cdot ; \theta)$ can be observed. On the basis of the observed sample values $t\left(x_{1}, x_{2}, \ldots, x_{n}\right)$ it is desired to estimate the value of the unknown parameter $\theta$ or some function $\tau(\theta)$ of the unknown parameter.

Let the value of some statistic, say $t\left(x_{1}, x_{2}, \ldots, x_{n}\right)$ represent, or estimate the unknown $\tau(\theta)$, such a statistic $t\left(x_{1}, x_{2}, \ldots, x_{n}\right)$ is called a point estimator [5].

The methods stated here like the least squares estimators (LSE) is commonly used in estimating parameters in linear regression models assuming a normal distribution of errors of observation and the condition of linear combination of the values with error variance being minimum. The maximum likelihood estimates (MLE) which yields an unbiased estimator, the method of moments, Bayesian estimators, minimum variance estimators $[6,7]$ and Cramer Rao lower bound [8] that is used to obtain the Uniformly Minimum variance unbiased estimator (UMVUE) are some of the techniques employed when estimating the unknown parameters.

\subsection{Methods of Finding Estimators}

Definition 1:

Any statistic whose function of observable random variables that is itself (a random variable) whose values are used to estimate $\tau(\theta)$ or $\theta$ where $\tau($.) is some function of the parameter $\theta$, is defined to be an estimator of $\tau(\theta)$ or $\theta$.

\subsubsection{Method of Moments}

One of the simplest methods of estimation is by moments 
$[11,12]$. With the Geeta distribution given as in (1), the $r^{\text {th }}$ moment of the population is defined as

$$
\mu_{r}^{\prime}=E\left(X^{r}\right)
$$

and the $j^{\text {th }}$ sample moment $M_{j}^{\prime}$ is defined by

$$
M_{j}^{\prime}=\frac{1}{n} \sum_{i=1}^{n} X_{i}^{j}
$$

Now from (11) we have:

$$
M_{1}^{\prime}=\frac{1}{n} \sum_{i=1}^{n} X_{i}=\bar{X} \text { and } M_{2}^{\prime}=\frac{1}{n} \sum_{i=1}^{n} X_{i}^{2}
$$

and from (10) we have:

$$
M_{1}^{\prime}=E(X)=\mu \text { and } M_{2}^{\prime}=E\left(X^{2}\right)=\operatorname{Var}(X)+\mu^{2}
$$

We see that (12) and (13) give

$$
M_{1}^{\prime}=\mu_{1}^{\prime}=\mu \text { and } M_{2}^{\prime}=\mu_{2}^{\prime}=\operatorname{Var}(X)+\mu^{2}(14)
$$

\subsubsection{Maximum Likelihood Estimator}

A maximum likelihood estimator, if it exists is the most efficient estimator; it happens sometimes that the method of maximum likelihood leads to complicated equations as we shall see in obtaining the estimate of parameter $\beta$ in Geeta distribution [9].

Let $x_{1}, x_{2}, \ldots, x_{n}$ be the values of a random sample $X_{1}, X_{2}, \cdots, X_{n}$ from $f_{X}(\cdot ; \theta)$

The likelihood function of $x_{1}, x_{2}, \ldots, x_{n}$ is given by;

$$
L(\underline{X}, \theta)=\prod_{i=1}^{n} f\left(x_{i} ; \theta\right)
$$

The maximum likelihood estimator of $\theta$ is obtained by taking the logs of the likelihood function, then differentiating partially with respect to $\theta$ and equate to zero,; that is;

$$
\hat{\theta}=\frac{\partial}{\partial \theta}[\ln \{L(\underline{X}, \theta)\}]=0
$$

\subsection{Properties of Estimators}

We have seen that many estimates will be available to us in any given situation. It is therefore desired to investigate some properties of point estimates. This will help in choosing the best estimates [7].

\subsubsection{Unbiasedness}

An estimator $T=t\left(x_{1}, x_{2}, \ldots, x_{n}\right)$ is defined to be unbiased estimator of $\tau(\theta)$ if and only if $E_{\theta}(T)=E_{\theta}\left[t\left(x_{1}, x_{2}, \cdots, x_{n}\right)\right]=\tau(\theta)$ or $E_{\theta}(T)=\tau(\theta)$ for all $\theta \in \Omega$.
An estimate that is not unbiased is called biased.

\subsubsection{Consistency}

Let $x_{1}, x_{2}, \ldots, x_{n}$ be a sequence of independent and identically distributed random variables with common density function $f_{X}(\cdot ; \theta) ; \theta \in \Omega$. A sequence of point estimates $T_{n}\left(x_{1}, x_{2}, \ldots, x_{n}\right)=T_{n}$ will be called consistent for $\theta$ if as $n \rightarrow \infty, E\left(T_{n}\right) \rightarrow \theta$ for each fixed $\theta \in \Omega$.

It is important to remember that consistency is essentially a large sample property.

If $T_{n}$ is a sequence of estimates such that $E\left(T_{n}\right) \rightarrow \theta$ and $\operatorname{Var}\left(T_{n}\right) \rightarrow 0$ as $n \rightarrow \infty$ then $T_{n}$ is consistent for $\theta$.

\subsubsection{Efficiency}

Let $T_{1}, T_{2}$ be two unbiased estimators for a parameter $\theta$. Suppose that $E_{\theta}\left(T_{1}^{2}\right)<\infty$ and $E_{\theta}\left(T_{2}^{2}\right)<\infty$, we define the efficiency of $T_{1}$ relative to $T_{2}$ by

$$
\operatorname{eff}_{\theta}\left(T_{1} / T_{2}\right)=\frac{\operatorname{Var}_{\theta}\left(T_{1}\right)}{\operatorname{Var}_{\theta}\left(T_{2}\right)}
$$

and say that $T_{1}$ is more efficient than $T_{2}$ if eff $\theta\left(T_{1} / T_{2}\right)<1$

Clearly the efficiency of the most efficient estimates is 1 , and the efficiency of any unbiased estimator $T_{1}$ is $>1$.

\subsubsection{Sufficiency}

Let $x_{1}, x_{2}, \ldots, x_{n}$ be a sequence of independent and identically distributed random variables with common density function $f_{X}(\cdot ; \theta) ; \theta \in \Omega$., where $\theta$ may be vector. A statistic $T=t\left(x_{1}, x_{2}, \ldots, x_{n}\right)$ is defined to be a sufficient statistic if and only if the conditional distribution of $\left(x_{1}, x_{2}, \ldots, x_{n}\right)$ given $T=t$ does not depend on $\theta$ for any value of $t$.

Example

Poisson sufficient statistic.

Let $X_{1}, X_{2}$ be independent Poisson variables with common expectation $\lambda$, so that their joint distribution is

$$
P\left(X_{1}=x_{1}, X_{2}=x_{2}\right)=\frac{\lambda^{x_{1}+x_{2}}}{x_{1} ! x_{2} !} e^{-2 \lambda}
$$

Then, the conditional distribution of $X_{1}$ given $X_{1}+X_{2}=t$ is given by

$$
\begin{aligned}
P\left(X_{1}\right. & \left.=x_{1} \mid X_{1}+X_{2}=t\right)=\frac{\lambda^{t} e^{-2 \lambda} / x_{1} !\left(t-x_{1}\right) !}{\sum_{y=0}^{t} \lambda^{t} e^{-2 \lambda} / y !(t-y) !} \\
& =\frac{1}{x_{1} !\left(t-x_{1}\right) !}\left(\frac{1}{\sum_{y=0}^{t} 1 / y !(t-y) !}\right)^{-1}
\end{aligned}
$$

Since this is independent of $\lambda$, so is the conditional distribution given $t$ of $\left(X_{1}, ., X_{2}=t-X_{1}\right)$, and hence $T=X_{1}$ $+X_{2}$ is a sufficient statistics for $\lambda$. To see how to reconstruct $\left(X_{1}, X_{2}\right)$ from $T$, note that 


$$
\sum \frac{1}{y !(t-y) !}=\frac{1}{t !} 2^{t}
$$

so that,

$$
P\left(X_{1}=x_{1} \mid X_{1}+X_{2}=t\right)=\left(\begin{array}{c}
t \\
x_{1}
\end{array}\right)\left(\begin{array}{l}
1 \\
2
\end{array}\right)^{x_{1}}\left(\begin{array}{l}
1 \\
2
\end{array}\right)^{t-x_{1}}
$$

that is, the conditional distribution of $X_{1}$ given $t$ is the binomial distribution $\mathrm{b}(1 / 2, t)$ corresponding to $t$ trials with success probability $1 / 2$. Let $X_{1}^{\prime}$ and $X_{2}^{\prime}=t-X_{1}^{\prime}$ be respectively the number of heads and the number of tails in $t$ tosses with a fair coin. Then, the joint conditional distribution of $\left(X_{1}^{\prime}, X_{2}^{\prime}\right)$ given $t$ is the same

as that of $\left(\mathrm{X}_{1}, X_{2}\right)$ given $t$.

\subsubsection{Completeness}

Let $x_{1}, x_{2}, \ldots, x_{n}$ denote a random sample from the density $f_{X}(\cdot ; \theta) ; \theta \in \Omega$ with parameter space $\Omega$ and let $T=t\left(x_{1}, x_{2}, \ldots, x_{n}\right)$ be a statistic. The family of densities of $T$ is defined to be complete if and only if $E[\mu(T)]=0$ for all $\theta \in \Omega$ implies that $\operatorname{Pr}[\mu(T)]=0$ for all $\theta \in \Omega$ or $\mu(T)=0$ for all $t$.

\section{Results and discussion}

\subsection{Results of Point Estimators}

The following results were obtained.

Theorem 1:

The point estimator of $\beta$ and $\theta$ using the method of moments is given by:

$\hat{\beta}=\frac{S^{2}-\bar{X}(\bar{X}-1)}{S^{2}-\bar{X}^{2}(\bar{X}-1)}$ and $\hat{\theta}=1-\frac{\bar{X}^{2}(\bar{X}-1)}{S^{2}}$, where

$S^{2}=\frac{\sum_{i=1}^{n}\left(X_{i}-\bar{X}\right)^{2}}{n-1}$ is the sample variance.

Proof:

From equations (2) and (14), we get

$$
\mu=\frac{1}{n} \sum_{i=1}^{n} X_{i}=\bar{X}
$$

which implies that $\hat{\mu}=\bar{X}$.

Hence from (15), we have

$$
\hat{\theta}=\frac{\bar{X}-1}{\bar{X} \hat{\beta}-1}
$$

Now from (12) and (14); we have

$$
M_{2}^{\prime}=\frac{1}{n} \sum_{i=1}^{n} X_{i}^{2}=\frac{\bar{X}(\bar{X}-1)(\hat{\beta} \bar{X}-1)}{\hat{\beta}-1}+\bar{X}^{2}
$$

which gives

$$
\hat{\beta}=\frac{\sum_{i=1}^{n} X_{i}^{2}-n \bar{X}^{2}-n \bar{X}^{2}+\frac{n \bar{X}^{2}}{n}}{\sum_{i=1}^{n} X_{i}^{2}-n \bar{X}^{2}+n \bar{X}^{2}+\frac{n \bar{X}^{2}}{n}}
$$

This further simplifies to

$$
\hat{\beta}=\frac{S^{2}-\bar{X}(\bar{X}-1)}{S^{2}-\bar{X}^{2}(\bar{X}-1)}
$$

where $S^{2}=\frac{\sum_{i=1}^{n}\left(X_{i}-\bar{X}\right)^{2}}{n-1}$ is the sample variance.

Using (16) in (18) we obtain the estimator of $\theta$ as

$$
\hat{\theta}=1-\frac{\bar{X}^{2}(\bar{X}-1)}{S^{2}}
$$

Theorem 2:

The maximum likelihood estimator of $\beta$ is given by the intersection of the two graphs $G(\beta)=\frac{(\beta-1) \bar{X}}{\beta \bar{X}-1}$ and $G(\beta)=e^{-H(\beta)}$ where $H(\beta)=\frac{1}{n \bar{X}} \sum_{i=2}^{k} \sum_{j=1}^{i} \frac{i \cdot f_{i}}{\beta X_{i}-j}$.

The maximum likelihood estimator of $\mu$ is given by

$$
\hat{\mu}=\bar{X} .
$$

Proof:

The Geeta distribution is as given in (1), and its likelihood function is given by

$$
L=\prod_{i=1}^{n} \frac{1}{\beta x-1}\left(\begin{array}{c}
\beta x-1 \\
x
\end{array}\right)\left(\frac{\mu-1}{\beta \mu-1}\right)^{\sum x_{i}-n}\left(\frac{\mu(\beta-1)}{\beta \mu-1}\right)^{\beta \sum x_{i}-\sum x_{i}}
$$

$$
=\left(\frac{\mu-1}{\beta \mu-1}\right)^{\sum x_{i}-n}\left(\frac{\mu(\beta-1)}{\beta \mu-1}\right)^{\beta \sum x_{i}-\sum x_{i}} \prod_{i=1}^{n} \frac{1}{\beta x_{i}-1}\left(\begin{array}{c}
\beta x_{i}-1 \\
x_{i}
\end{array}\right)
$$

Taking the logs of the likelihood function, and partially differentiating with respect to $\mu$ and equating to zero we have

$$
\hat{\mu}=\bar{X}
$$

Differentiating the log-likelihood function (20) partially with respect to $\beta$ and equating the results to zero, we have 


$$
\begin{aligned}
& -\mu\left(\sum X_{i}-n\right)+\sum X_{i} \log [\mu(\beta-1)] \\
& +\frac{\mu \beta \sum X_{i}}{\mu(\beta-1)}-\frac{\mu \sum X_{i}}{\mu(\beta-1)}-\sum X_{i} \log (\mu \beta-1)+ \\
& \frac{\mu \beta \sum X_{i}}{\mu \beta-1}-\frac{\mu \sum X_{i}}{\mu \beta-1}-\frac{X_{i}}{\beta X_{i}-1}-\frac{X_{i}}{\beta X_{i}-1}
\end{aligned}
$$

which further simplifies to

$$
\begin{aligned}
& \log [\bar{X}(\beta-1)]-\log [\bar{X} \beta-1]= \\
& \frac{\beta}{\beta-1}-\frac{1}{\beta-1}-\frac{\bar{X}}{\beta \bar{X}-1}+\frac{\beta \bar{X}}{\beta \bar{X}-1} \\
& +\frac{\bar{X}-1}{\beta \bar{X}-1}+\sum_{i=1}^{n} \frac{X_{i}}{\beta X_{i}-1}
\end{aligned}
$$

Therefore

$$
\log \left[\frac{\bar{X}(\beta-1)}{\bar{X} \beta-1}\right]=\sum_{i=1}^{n} \frac{X_{i}}{\beta X_{i}-1}
$$

which implies that

$$
\frac{\bar{X}(\beta-1)}{\bar{X} \beta-1}=e^{\sum_{i=1}^{n} \frac{X_{i}}{\beta X_{i}-1}}
$$

which can be re-written as

$$
\frac{\bar{X}(\beta-1)}{\bar{X} \beta-1}=e^{H(\beta)}=G(\beta)
$$

where

$$
\begin{gathered}
f_{1}+f_{2}+\cdots+f_{k}=n \text { and } \\
\sum_{i=1}^{k} \frac{i \cdot f_{i}}{n}=\frac{1 \cdot f_{1}+2 \cdot f_{2}+\cdots+k \cdot f_{k}}{n}=\bar{X}
\end{gathered}
$$

such that

$$
H(\beta)=\frac{1}{n \bar{X}} \sum_{i=2}^{k} \sum_{j}^{i} \frac{i \cdot f_{i}}{\beta X_{i}-j}
$$

The equation (26) cannot be specifically solved for the maximum likelihood estimator of $\beta$. However, the function

$$
G(\beta)=\frac{(\beta-1) \bar{X}}{\beta \bar{X}-1}
$$

does monotonically increase from 0 to 1 as $\beta$ increases from 1 to $\infty$. The function $H(\beta)$ is a sum of finite number of functions. Each one of these functions monotonically decreases as $\beta$ increases from 1 to $\infty$. Therefore the function $H(\beta)$ monotonically decreases with the increase in the value of $\beta$. The two graphs (curves), that is

$$
G(\beta)=\frac{(\beta-1) \bar{X}}{\beta \bar{X}-1} \text { and } G(\beta)=e^{H(\beta)}
$$

when drawn, they are both monotonically increasing and have a single point of intersection. Therefore the value of $\beta$ given by this unique point of intersection is the maximum likelihood estimator of $\beta$.

\subsection{Properties of the Point Estimators}

We have seen that many estimates will be available to us in any given situation. It is therefore desired to investigate some properties of point estimates. This will help in choosing the best estimates. The following results of the properties of the various point estimators were obtained.

Theorem 3

$$
\hat{\theta}=1-\frac{\bar{X}^{2}(\bar{X}-1)}{S^{2}} \text { is an unbiased estimator of } \theta \text {. }
$$

Proof

We need to show that $E(\hat{\theta})=\theta$

Assuming that $\bar{X}$ and $S^{2}$ are independent and using equations (2) and (3); we can show that $E(\hat{\theta})=g(\theta)$.

Theorem 4

$T=\sum_{i=1}^{n} X_{i}$ is sufficient statistic for $\theta$

Proof:

The conditional distribution is given by

$$
f\left(\left(x_{1}, x_{2}, \cdots, x_{n}\right) / t\right)=f(\underline{x} / t)=\frac{f(\underline{x}, t)}{f(t)}
$$

but

$$
f\left(x_{1}, x_{2}, \cdots, x_{n}\right)=f\left(x_{1}, x_{2}, \cdots, x_{n-1}, t\right)
$$

therefore

$$
f(\underline{x}, t)=f\left(x_{1}, x_{2}, \cdots, x_{n-1}, t\right)=\prod_{i=1}^{n-1} f\left(x_{i}\right) f(t)
$$

which further simplifies to

$$
f(\underline{x}, t)=\theta^{t-n}(1-\theta)^{\beta t-t} \prod_{i=1}^{n-1} \frac{1}{\beta x_{i}-1}
$$

but

$$
\begin{aligned}
& f(t)=\operatorname{Pr}(T=t)=\operatorname{Pr}\left(\sum_{i=1}^{n} x_{i}=t\right) \\
& =\frac{n}{\beta t-1}\left(\begin{array}{c}
\beta t-1 \\
t
\end{array}\right) \theta^{t-n}(1-\theta)^{\beta t-t}
\end{aligned}
$$


Therefore

$$
f(x / t)=\frac{\theta^{t-n}(1-\theta)^{\beta t-t} \prod_{i=1}^{n-1} \frac{1}{\beta x_{i}-1}}{\theta^{t-n}(1-\theta)^{\beta t-t}\left(\begin{array}{c}
\beta t-1 \\
t
\end{array}\right)}
$$

which then gives

$$
f(x / t)=\frac{1}{\left(\begin{array}{c}
\beta t-1 \\
t
\end{array}\right)} \prod_{i=1}^{n-1} \frac{1}{\beta x_{i}-1}
$$

Therefore the conditional distribution of $\left(x_{1}, x_{2}, \cdots, x_{n}\right)$ given $t$ does not depend on $\theta$, hence $T=\sum_{i=1}^{n} X_{i}$ is sufficient statistic for $\theta$.

Theorem 5

$T=\sum_{i=1}^{n} X_{i}$ is a complete statistic.

Proof:

$T=\sum_{i=1}^{n} X_{i}$ is a complete statistic if $E[\mu(T)]=0$.

The family of Geeta distribution densities is given by (1). The Geeta probability model belongs to the exponential family, is a complete family

Let

$$
\operatorname{Pr}\left(T=t=\sum_{i=1}^{n} x_{i}\right)=\left(\begin{array}{c}
\beta t-1 \\
t
\end{array}\right) \theta^{t-n}(1-\theta)^{\beta t-t}
$$

and $\mu(T)$ be any function of $t$ such that $E[\mu(T)]=0$ for all $\theta \in \Omega$ implies that $\mu(T)=0$ for all $t$.

Therefore

$$
\begin{aligned}
& E(\mu(t))=\sum_{t=1}^{n} \mu(t)\left(\begin{array}{c}
\beta t-1 \\
t
\end{array}\right) \theta^{t-n}(1-\theta)^{\beta t-t} \\
& =\sum_{t=1}^{n} \varphi(t) \theta^{t-n}(1-\theta)^{\beta t-t}
\end{aligned}
$$

where $\varphi(t)=\mu(t)\left(\begin{array}{c}\beta t-1 \\ t\end{array}\right)$

which can further be expressed as

$$
\begin{aligned}
& E(\mu(t))=\sum_{t=1}^{n} \varphi(t) \theta^{t-n}(1-\theta)^{\beta t-t} \\
& =\varphi(1) \theta^{1-n}(1-\theta)^{\beta-1}+\cdots \\
& +\varphi(n) \theta^{n-n}(1-\theta)^{n(\beta-1)}
\end{aligned}
$$

This is a polynomial of degree $n$ in $\theta(1-\theta)^{\beta-1}$ and is therefore a zero polynomial. That is

$$
\varphi(1)=\varphi(2)=\cdots=\varphi(n)=0
$$

and $\varphi(t)=0$ for all $t=1,2, \cdots, n$

but

$$
\varphi(t)=\mu(t)\left(\begin{array}{c}
\beta t-1 \\
t
\end{array}\right)=0
$$

Since $\left(\begin{array}{c}\beta t-1 \\ t\end{array}\right) \neq 0$ then $\mu(t)=0$ for all $t$.

Therefore

$$
U=\left[\left(\begin{array}{c}
\beta t-1 \\
t
\end{array}\right) \theta^{t-n}(1-\theta)^{\beta t-t}, 0<\theta<1\right]
$$

is a complete family. Hence $T$ is a complete and sufficient statistic.

\section{Conclusion}

In most branches of knowledge, experiments are a way of life and in probability and statistics, there are special types of experiments which are in practice the distribution models of either discrete or continuous distribution. The distribution models are applied in manufacturing industries, and the medicine world, and it has eased the handling of large data sets. The Geeta distribution is being used because of the presence of the unknown constant. These unknown parameters are the most vital part of the distribution, because of the information it contains.

Geeta distribution and its properties were reviewed and discussed and it proves that it is a discrete distribution with sum of all probabilities is unity. It is a preferred distribution model to be used because of its relevance in modern research and application in genetics, sales and fashion industry, branching processes (queue) and bunching models.

Therefore, the properties and characteristics the Geeta distribution satisfy and meet the conditions of any probability distribution, that is sum of all probabilities for all values of a random variable $x$ is unity. The mean, variance, moment generating function, probability generating function and the central theorem exist [10]. The estimators of the parameters were obtained and the properties of a good estimator such unbiasedness, sufficiency and completeness were proven to hold.

\section{References}

[1] Hogg, R. V. Craig, A. T. (1956): Introduction to mathematical statistics. Pg. 200-227.

[2] E. L. Lehmann, George Casella (1998), Theory of Point Estimation, $2^{\text {nd }}$ Ed., Springer-Verlag New York, Inc. 
[3] Consul, P. C. (1990a), Geeta distribution and is Properties, Communication in Statistics-Theory and Methods, 19, 30513068[7.2.4].

[4] Consul, P. C (1990b), New Class of Location -Parameter Discrete Probability distribution and their characteristics. Communication in Statistics-Theory and methods, 19, 46534666. [2.2.2, 7.2.4].

[5] Harold J. Larson (1934), Statistics: An introduction to Statistics. Pg. 171-209.

[6] Robert Bassett, Julio Deride (2016), Maximum a posteriori estimators as a limit of Bayes estimators, Journal of Mathematical Programming.

[7] Gupta S. P. (2016), Statistical Methods, Sultan Chand \& Sons, 43rd Ed.
[8] H. Cramer (1946), Mathematical Methods of statistics, Princeton University Press.

[9] Paul Vos and Qiang Wu (2015), Maximum likelihood estimators uniformly minimize distribution variance among distribution unbiased estimators in exponential families, Bernoulli 21(4), Pg. 2120-2138.

[10] Rohatgi, V. K. (1975), An introduction to probability theory and mathematical statistics. Pg. 337-401.

[11] Mood, A. N. Gray bill, F. A and Boes, D. C. (1963): Introduction to the theory of statistics. Pg. 271-357. Saxena, H. C. Surendran, P. U. (1967) statistical Inference. Pg. 37-57. 